\title{
An extension of compromise ranking method with interval numbers for the evaluation of renewable energy sources
}

\author{
M. Mousavi ${ }^{a^{*}}$ and R. Tavakkoli-Moghaddam ${ }^{\mathrm{b}}$
}

${ }^{a}$ Economics Faculty, University of Tehran, Tehran, Iran

${ }^{b}$ School of Industrial Engineering, College of Engineering, University of Tehran, Tehran, Iran

\section{H R O N I C L E}

Article history:

Received December 2, 2013

Accepted February 20, 2014

Available online

May 212014

Keywords:

Renewable energy source

Evaluation problem

Multi-attribute decision making

Compromise ranking

\section{A B S T R A C T}

Evaluating and prioritizing appropriate renewable energy sources is inevitably a complex decision process. Various information and conflicting attributes should be taken into account. For this purpose, multi-attribute decision making (MADM) methods can assist managers or decision makers in formulating renewable energy sources priorities by considering important objective and attributes. In this paper, a new extension of compromise ranking method with interval numbers is presented for the prioritization of renewable energy sources that is based on the performance similarity of alternatives to ideal solutions. To demonstrate the applicability of the proposed decision method, an application example is provided and the computational results are analyzed. Results illustrate that the presented method is viable in solving the evaluation and prioritization problem of renewable energy sources.

\section{Introduction}

Recently, renewable energy sources have been considered in developing countries in order to reduce environmental pollutions, substantially; however, these sources may increase high financial risks. The new decision making methods can be useful for policy makers and energy planners to take various policy criteria into account and appraise the necessary plans for long term planning based on the most preferred energy options (Diakoulaki \& Karangelis, 2007; Pao \& Fu, 2013; Cannemi et al., 2014). The prioritization and selection of the best renewable energy sources depends on different attributes (factors) and preferences provided by policy makers and energy planners. In fact, the decision problem of the renewable energy sources can be made based on numerous measures, which may be in conflict. Hence, the prioritization and selection problem can be solved by multiple attributes decisionmaking (MADM). The MADM is a well-known approach for the decision-making, which often takes multiple and conflicting objectives into consideration. Generally, it has aimed to arrange all the best criteria values achievable, while the worst solution is arranged by all the worst attribute values achievable.

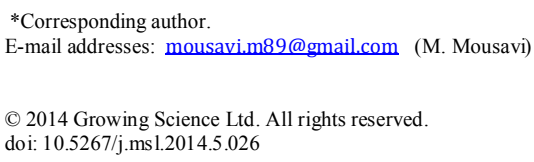


For the literature review on the prioritization of renewable energy sources, Diakoulaki and Karangelis (2007) evaluated various scenarios for the development of power generation sector in Greece by the MADM and cost-benefit analysis approaches. Loken (2007) applied MADM methods for energy planning problems and tried to choose the optimal solution. Banos et al. (2011) reported the optimization methods, which could be employed in the sustainable energy. Iakovou et al. (2010) focused on the related field of energy sources from waste biomass. They aimed to recognize the barriers faced by that industry. Wang et al. (2010) appraised coal, petroleum, natural gas, nuclear energy and renewable energy resources as energy options for China by employing a hierarchical decision model. The computational results illustrated that the coal and renewable energy could be regarded as the major preferred energy options.

Akash et al. (1999) compared electricity power production options in Jordan by implementing analytical hierarchy process (AHP). Erol and Kilk1s (2012) used the AHP to evaluate energy source policy as a challenging issue for the managers. They tried to derive sustainable, robust, and long-term energy policies, and then the method was implemented in the district of Aydin in Turkey. San Cristóbal (2011) considered the MADM in the selection of renewable energy projects, and then it was utilized for in the renewable energy projects corresponding to the renewable energy plan launched by the Spanish government. The computational results indicated that the biomass plant option, cocombustion in a conventional power plant is the best option, followed by the wind power and solar thermo-electric options. Based on the gap in the related literature, this paper presents a new decision approach for the prioritization of renewable energy sources by considering various conflicting assessment attributes. This approach is based on a new extension of the compromise ranking method with interval numbers that has good potential for solving evaluation problem and also has the following characteristics:

- The method considers an unlimited range of renewable energy properties, by the concepts of compromise programming and interval computations and by regarding several system attributes/components;

- It maintains a simple computation process and easily programmed; and

- It proposes an output as a preferential ranking of the renewable energy alternatives with numerical values in interval form to figure out the differences and similarities between alternatives.

The rest of this paper is organized as follows. The MADM methods are described in section 2. The proposed decision approach is explained in section 3. Section 4 presents the detail of the proposed approach through an application example for renewable energy sources, and discussion of results is also given. Finally, conclusion is given in section 5.

\section{Multiple attributes decision-making (MADM)}

The MADM is applied in order to evaluate, to rank or to select a set of options (alternatives) under usually independent and conflicting criteria (Hwang \& Yoon, 1981). The approach often needs decision makers (DMs) or experts to obtain qualitative and/ or quantitative evaluations for establishing the performance of each alternative versus each attribute, and the relative importance of evaluation attribute with respect to the overall goal. In general, the MADM includes the following common features:

- a set of comparable alternatives;

- multiple attributes for appraising the alternatives;

- non-commensurable units for calculating the performance of the alternatives on each attribute; and

- attributes weights for considering the relative importance of each attribute. 
The MADM is often utilized to solve various complex decision making and/or selection problems (e.g., Loken, 2007; Mojtahedi et al., 2010; Ebrahimnejad et al., 2010; San Cristóbal 2011). One of the most popular MADM methods is compromise solution-based methods, like compromise ranking method, which has been originally proposed by Opricovic and Tzeng (2004). The fundamental concept in this method is that the alternative should have the distance from the ideal solution as short as possible and the distance from the negative-ideal solution as far as possible concurrently. The positive ideal solution is defined as the alternative with the highest value while the negative ideal solution is defined as the one with the least test value. The compromise solution is regarded as a feasible solution, in which a compromise means an agreement determined by mutual concessions. The method is followed by that of improved work efficiency and promoted reactions.

In the last decade, the compromise solution-based methods have been applied to many different fields. Consequently, the attractive results have been reported in the literature; for instance, risk evaluation problems (Ebrahimnejad et al., 2010), contractor selection problems (Vahdani et al., 2013), and aircraft selection problems (Yeh and Chang, 2009).

\section{Proposed approach}

In this section, a new approach is presented for the prioritization of renewable energy sources. This approach based on compromise ranking method copes with scalar values that take into account both the best and worst alternatives concurrently. The decision approach is designed to evaluate and rank renewable energy sources versus multiple conflicting criteria or factors. The steps of the proposed decision approach are provided below.

Step 1. A group of experts or DMs is formed for renewable energy sources.

Step 2. Renewable energy data is obtained for the evaluation and prioritization problem.

Step 2-1. Renewable energy evaluation problem is defined for creating the list of renewable energy alternatives and attributes.

Step 2-2. Renewable energy data are collected by historical information, other documents and group decision techniques, such as Brainstorming, Delphi and NGT.

Step 2-3. A list of renewable energy alternatives and attributes is provided for the evaluation and prioritization.

Step 3. Proposed compromise ranking method is performed for evaluating and prioritizing the renewable energy sources.

Step 3-1. The linguistic terms and/or interval numbers $\left(x_{i j}^{L}, x_{i j}^{U} i=1,2, \ldots, m, j=1,2, \ldots, n\right)$ for renewable energy sources versus selected attributes are provided, and then the appropriate linguistic terms and/interval numbers to the weight of the attributes $\left(w_{j}^{L}, w_{j}^{U}, j=1,2, \ldots, n\right)$ are assigned.

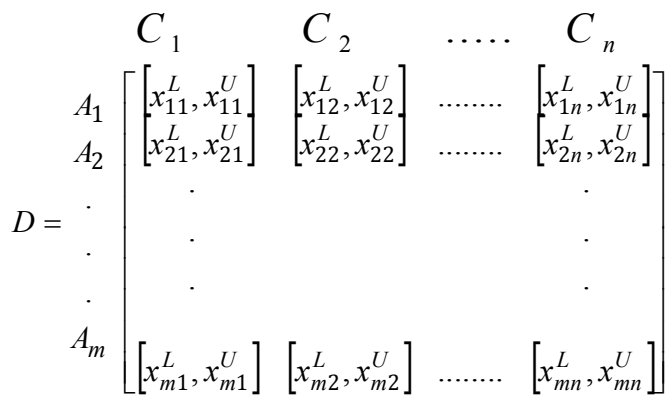


Step 3-2. The normalized decision matrix with interval numbers is established by the following equations:

$$
\begin{aligned}
& f_{i j}{ }^{L}=x_{i j}{ }^{L} / \sqrt{\sum_{j=1}^{m}\left(x_{i j}{ }^{L}\right)^{2}+\left(x_{i j}{ }^{U}\right)^{2}}, j=1,2, \ldots, m, \quad i=1,2, \ldots n, \\
& f_{i j}^{U}=x_{i j}^{U} / \sqrt{\sum_{j=1}^{m}\left(x_{i j}{ }^{L}\right)^{2}+\left(x_{i j}{ }^{U}\right)^{2}}, j=1,2, \ldots, m, i=1,2, \ldots n .
\end{aligned}
$$

Step 3-3. The best and worst values are determined, known as positive ideal and negative ideal solutions, $(j=1,2, \ldots, n)$.

$$
\begin{aligned}
& f_{j}^{*}=\left\{\left(\max _{i} f_{i j}^{U} \mid j \in B\right) \operatorname{or}\left(\min _{i} f_{i j}^{L} \mid j \in C\right)\right\}, \\
& f_{j}^{-}=\left\{\left(\min _{i} f_{i j}^{L} \mid j \in B\right) \operatorname{or}\left(\max _{i} f_{i j}^{U} \mid j \in C\right)\right\},
\end{aligned}
$$

where, $B$ is associated with benefit attributes, and $C$ is associated with cost attributes.

Step 3-4. $S_{i}$ and $R_{i}$ values in interval forms are computed by the following equations, $(i=1,2$, $\ldots, m)$.

$$
\begin{aligned}
& S_{i}^{L}=\sum_{j \in B} w_{j}^{L}\left(f_{j}^{*}-f_{i j}^{U}\right) /\left(f_{j}^{*}-f_{j}^{-}\right)+\sum_{j \in C} w_{j}^{L}\left(f_{i j}^{L}-f_{j}^{*}\right) /\left(f_{j}^{-}-f_{j}^{*}\right) \\
& S_{i}^{U}=\sum_{j \in B} w_{j}^{U}\left(f_{j}^{*}-f_{i j}^{L}\right) /\left(f_{j}^{*}-f_{j}^{-}\right)+\sum_{j \in C} w_{j}^{U}\left(f_{i j}^{U}-f_{j}^{*}\right) /\left(f_{j}^{-}-f_{j}^{*}\right) \\
& R_{i}^{L}=\max _{j}\left\{w_{j}^{L}\left(f_{j}^{*}-f_{i j}^{U}\right) /\left(f_{j}^{*}-f_{j}^{-}\right)\left|j \in B, w_{j}^{L}\left(f_{i j}^{L}-f_{j}^{*}\right) /\left(f_{j}^{-}-f_{j}^{*}\right)\right| j \in C\right\} \\
& R_{i}^{U}=\max _{j}\left\{w_{j}^{U}\left(f_{j}^{*}-f_{i j}^{L}\right) /\left(f_{j}^{*}-f_{j}^{-}\right)\left|j \in B, w_{j}^{U}\left(f_{i j}^{U}-f_{j}^{*}\right) /\left(f_{j}^{-}-f_{j}^{*}\right)\right| j \in C\right\}
\end{aligned}
$$

where, $S_{i}$ is $A_{i}$ versus the attributes provided by the sum of the distance for the best value, and $R_{i}$ is $A_{i}$ versus the $j$-th factor or attribute, provided by the maximum distance from the worst value. Moreover, $w_{j}$ is the weight of attribute regarding the group of the DMs' preferences as the relative importance of attributes.

Step 3.5. The following values for ranking index are calculated:

$$
\begin{gathered}
S^{*}=\min _{i} S_{i}^{L} \quad S^{-}=\max _{i} S_{i}^{U} \quad R^{*}=\min _{i} R_{i}^{L} \quad R^{-}=\max _{i} R_{i}^{U} \\
Q_{i}^{L}=\left(\left|S_{i}^{L}-S^{*}\right| /\left(\left|S_{i}^{L}-S^{*}\right|+\left|R_{i}^{L}-R^{*}\right|\right)\right)\left(\left(S_{i}^{L}-S^{*}\right) /\left(S^{-}-S^{*}\right)\right)+ \\
\quad\left(1-\left|S_{i}^{L}-S^{*}\right| /\left(\left|S_{i}^{L}-S^{*}\right|+\left|R_{i}^{L}-R^{*}\right|\right)\right)\left(\left(R_{i}^{L}-R^{*}\right) /\left(R^{-}-R^{*}\right)\right) \\
\left.Q_{i}^{U}=\left(\left|S_{i}^{U}-S^{*}\right| /\left(\left|S_{i}^{U}-S^{*}\right|+\left|R_{i}^{U}-R^{*}\right|\right)\right)\left(S_{i}^{U}-S^{*}\right) /\left(S^{-}-S^{*}\right)\right)+ \\
\quad\left(1-\left|S_{i}^{U}-S^{*}\right| /\left(\left|S_{i}^{U}-S^{*}\right|+\left|R_{i}^{U}-R^{*}\right|\right)\right)\left(\left(R_{i}^{U}-R^{*}\right) /\left(R^{-}-R^{*}\right)\right)
\end{gathered}
$$

$S^{*}$ is regarded as the minimum value of $S_{i}$, which is the maximum majority rule. In addition, $R^{*}$ is regarded as the minimum value of $R_{i}$, which is the minimum individual regret of the opponent. Hence, the proposed index $Q_{i}=\left[Q_{i}^{L}, Q_{i}^{U}\right]$ is presented based on the consideration of both the group utility and the individual regret of the opponent. 
Step 3.6. Final $Q_{i}$ values are calculated by the following equation:

$$
Q_{i}=\left(Q_{i}^{L}+Q_{i}^{U}\right) / 2
$$

Step 4. The renewable energy sources as potential alternatives are sorted by the value $Q_{i}$ in a decreasing order.

\section{Application example}

This section illustrates the proposed approach through an application example for the prioritization of renewable energy sources.

\subsection{Computational results}

The proposed approach is elaborated for the prioritization of renewable energy sources. For this purpose, a required decision committee of experts or DMs in energy planning is formed. The team establishment step is needed to consider the different conflicting attributes with respect to the main goals for the energy planning. To gather the renewable energy sources data, we utilize historical information, energy project records, documents and the literature review regarding energy assessment. The renewable energy sources are listed as below:

\section{Biomass (A1) Geothermal (A2) Hydropower (A3) Solar (A4) Wind (A5)}

In the following, the assessment attributes in energy planning can be provided as follows:

- Technological factor (C1): The factor assesses the technology of the renewable energy sources,

- Environmental factor (C2): The factor is related to environmental pollution emissions,

- Social factor $(\mathrm{C} 3)$ : The factor tries to take account of the reactions from the interest of social groups regarding renewable energy options,

- Economical factor (C4): The factor is related to engineering economics attribute, like NPW and IRR.

Table 1 in 7-scale is given to determine the weights of attributes and the performance ratings of the decision matrix provided by experts as below:

\section{Table 1}

7-scale for the weights of attributes and the performance ratings

\begin{tabular}{|c|c|c|}
\hline Scale & Description & Interval numbers \\
\hline Very Good/ Very High & The performance rating (or the weight of attribute) is very good (very high). & {$[85,100]$} \\
\hline Good/ High & The performance rating (or the weight of attribute) is good (high). & {$[70,85]$} \\
\hline Medium Good/ Medium High & $\begin{array}{l}\text { The performance rating (or the weight of attribute) is medium good (medium } \\
\text { high). }\end{array}$ & {$[55,70]$} \\
\hline Fair & The performance rating (or the weight of attribute) is fair. & {$[40,55]$} \\
\hline Medium Poor / Medium Low & $\begin{array}{l}\text { The performance rating (or the weight of attribute) is medium poor (medium } \\
\text { low). }\end{array}$ & {$[25,40]$} \\
\hline Poor / Low & The performance rating (or the weight of attribute) is poor (low). & {$[10,25]$} \\
\hline Very Poor / Very Low & The performance rating (or the weight of attribute) is very poor (very low). & {$[0,10]$} \\
\hline
\end{tabular}

Based on the Table 1, the experts' judgments regarding the weights of attributes are provided for the prioritization of renewable energy sources in Table 2. 
Table 2

Weights of four attributes obtained by the experts' judgments

\begin{tabular}{llllllll}
\hline Attributes & VL & L & ML & F & MH & H & VH \\
\hline Technological & $\square$ & $\square$ & $\square$ & $\square$ & $\square$ & & $\square$ \\
Environmental & $\square$ & $\square$ & $\square$ & $\square$ & & $\square$ & $\square$ \\
Social & $\square$ & $\square$ & $\square$ & $\square$ & & $\square$ & $\square$ \\
Economical & $\square$ & $\square$ & $\square$ & $\square$ & $\square$ & $\square$ & $\square$ \\
\hline
\end{tabular}

The experts' judgments based on the Table 1 for the performance ratings of renewable energy sources as a decision matrix in interval form are provided in Table 3.

Table 3

Performance rating of five renewable energy sources

\begin{tabular}{ccccc}
\hline $\begin{array}{c}\text { Renewable energy } \\
\text { sources }\end{array}$ & $\begin{array}{c}\text { Technological } \\
(\mathrm{C} 1)\end{array}$ & $\begin{array}{c}\text { Environmental } \\
(\mathrm{C} 2)\end{array}$ & $\begin{array}{c}\text { Social } \\
(\mathrm{C} 3)\end{array}$ & $\begin{array}{c}\text { Economical } \\
(\mathrm{C} 4)\end{array}$ \\
\hline Biomass (A1) & $\mathrm{MH}$ & $\mathrm{L}$ & $\mathrm{F}$ & $\mathrm{MH}$ \\
Geothermal (A2) & $\mathrm{MH}$ & $\mathrm{ML}$ & $\mathrm{F}$ & $\mathrm{MH}$ \\
Hydropower (A3) & $\mathrm{MH}$ & $\mathrm{L}$ & $\mathrm{H}$ & $\mathrm{H}$ \\
Solar (A4) & $\mathrm{H}$ & $\mathrm{VL}$ & $\mathrm{H}$ & $\mathrm{VH}$ \\
Wind (A5) & $\mathrm{H}$ & $\mathrm{VL}$ & $\mathrm{MH}$ & $\mathrm{VH}$ \\
\hline
\end{tabular}

To solve the energy prioritization problem according to steps of the proposed compromise ranking method with interval numbers, the renewable energy sources are evaluated and ranked in the application example. Normalized decision matrix with interval numbers is given in Table 4. Then, the values of $S_{i}$ and $R_{i}$ are calculated and provided in Table 5. Finally, rating of $Q_{i}$ in interval numbers and ranking of renewable energy sources are reported in Table 6.

Table 4.

Normalized decision matrix with interval numbers

\begin{tabular}{ccccc}
\hline Renewable energy sources & Technological (C1) & Environmental (C2) & Social (C3) & Economical (C4) \\
\hline Biomass (A1) & {$[0.251,0.319]$} & {$[0.161,0.402]$} & {$[0.197,0.270]$} & {$[0.220,0.280]$} \\
Geothermal (A2) & {$[0.251,0.319]$} & {$[0.402,0.643]$} & {$[0.197,0.270]$} & {$[0.220,0.280]$} \\
Hydropower (A3) & {$[0.251,0.319]$} & {$[0.161,0.402]$} & {$[0.344,0.418]$} & {$[0.280,0.340]$} \\
\hline Solar (A4) & {$[0.319,0.388]$} & {$[0.000,0.161]$} & {$[0.344,0.418]$} & {$[0.340,0.400]$} \\
Wind (A5) & {$[0.319,0.388]$} & {$[0.000,0.161]$} & {$[0.270,0.344]$} & {$[0.340,0.400]$} \\
\hline
\end{tabular}

Table 5

Values of $S_{i}$ and $R_{i}$

\begin{tabular}{ccc}
\hline Renewable energy sources & $S_{i}$ & $R_{i}$ \\
\hline Biomass (A1) & {$[132.083,283.750]$} & {$[46.667,85.000]$} \\
Geothermal (A2) & {$[152.708,310.000]$} & {$[46.667,85.000]$} \\
Hydropower (A3) & {$[72.083,208.750]$} & {$[35.000,85.000]$} \\
Solar (A4) & {$[0.000,111.667]$} & {$[0.000,42.500]$} \\
Wind (A5) & {$[18.333,135.000]$} & {$[18.333,46.667]$} \\
\hline
\end{tabular}


Table 6

Rating of $Q_{i}$ and ranking of renewable energy sources

\begin{tabular}{ccc}
\hline Renewable energy sources & $O_{i}$ & Rank \\
\hline Biomass (A1) & {$[0.458,0.935]$} & 4 \\
Geothermal (A2) & {$[0.506,1.000]$} & 5 \\
Hydropower (A3) & {$[0.291,0.768]$} & 3 \\
Solar (A4) & {$[0.000,0.399]$} & 1 \\
Wind (A5) & {$[0.137,0.465]$} & 2 \\
\hline
\end{tabular}

According to the computational results for the energy prioritization problem by the proposed compromise ranking method with interval numbers, final rankings of renewable energy sources are provided as follows:

\section{Solar (A4) 2. Wind (A5) 3. Hydropower (A3) 4. Biomass (A1) 5.Geothermal (A2)}

\subsection{Discussion of results}

In this sub-section, the sensitivity analysis is provided for the weight of the majority attributes in the proposed compromise ranking method with interval numbers. The ranking index is considered and reported in Table 7. According to this table, the computational results indicate what the experts or DMs expect. It means that rankings of the renewable energy sources have enough stability.

Table 7

Values effects of the majority attributes for the ranking of renewable energy sources

\begin{tabular}{cccccc}
\hline & \multicolumn{5}{c}{ Majority attributes } \\
\cline { 2 - 6 } Renewable energy sources & 0 & 0.3 & 0.5 & 0.7 & 1 \\
\hline Biomass (A1) & 0.775 & 0.743 & 0.723 & 0.702 & 0.671 \\
Geothermal (A2) & 0.775 & 0.766 & 0.760 & 0.755 & 0.746 \\
Hydropower (A3) & 0.706 & 0.630 & 0.579 & 0.529 & 0.453 \\
Solar (A4) & 0.250 & 0.229 & 0.215 & 0.201 & 0.180 \\
Wind (A5) & 0.382 & 0.342 & 0.315 & 0.288 & 0.247 \\
\hline
\end{tabular}

The computational results have highlighted that the proposed compromise ranking method with interval numbers takes account of the requirements as well as the weights of attributes and evaluates the renewable energy sources versus the ideal reference points. Hence, it is a suitable and helpful method through the multi-attributes analysis for the real-life energy applications.

\section{Conclusions}

In current situations of global changes and development in the field of environment protection and due to perishable nature of the fossil resources, using renewable energy has accelerated trend. In order to fulfill the objectives of renewable energies use in developing countries, different factors or attributes can be categorized as technological, environmental, social and economic factors. A new decision approach with interval numbers is proposed for prioritizing renewable energies based on the compromise ranking method. In this method, different energy candidates are evaluated with respect to the energy assessment attributes. It is an efficient method in ranking renewable energy sources as it takes into account the alternatives distances from an ideal solution. This method also has the advantages of considering both qualitative and quantitative conflicting attributes concurrently. An application example was presented to demonstrate the suitability of the proposed decision approach with interval numbers. For the purpose of the calculation, ranking renewable energy alternatives is completed based on compromise solution concept and interval computations followed by sensitivity 
analysis. Computational results demonstrated that the effectiveness of the presented decision approach for the prioritization problem of the renewable energy sources. It can support top managers and policy makers to properly identify and appraise the renewable energy sources in developing countries. For the further research, it will be interesting to employ the proposed approach through a decision support system to facilitate the prioritization problem of the renewable energy sources.

\section{References}

Akash, B.A., Mamlook, R., Mohsen, M.S., (1999). Multi-criteria selection of electric power plants using analytical hierarchy process. Electric Power Systems Research, 52, 29-35.

Baños, R., Manzano-Agugliaro, F., Montoya, F.G., Gil, C., Alcayde, A, Gómez, J., (2011). Optimisation methods applied to renewable and sustainable energy: a review. Renewable and Sustainable Energy Reviews, 15, 1753-1766.

Cannemi,M., García-Melón, M., Aragonés-Beltrán, P. \& Gómez-Navarro, T. (2014). Modeling decision making as a support tool for policy making on renewable energy development, Energy Policy, 67, 127-137.

Diakoulaki, D. \& Karangelis, F., (2007). Multi-criteria decision analysis and cost-benefit analysis of alternative scenarios for the power generation sector in Greece, Renewable and Sustainable Energy Reviews, 11, 716-727.

Ebrahimnejad, S., Mousavi, S.M., \& Seyrafianpour, H., (2010). Risk identification and assessment for build-operate-transfer projects: a fuzzy multi attribute decision making model, Expert Systems With Applications, 37, 575-586.

Erol, O., \& Kilkıs, B., (2012). An energy source policy assessment using analytical hierarchy process, Energy Conversion and Management, 63, 245-252.

Hwang, C. L., \& Yoon, N. (1981). Multiple attributes decision making methods and application. Berlin: Springer-Verlag.

Iakovou, E., Karagiannidis, A., Vlachos, D., Toka, A., Malamakis, A. (2010). Waste biomass toenergy supply chain management: a critical synthesis. Waste Management, 30, 1860-1870.

Loken, E., (2007). Use of multicriteria decision analysis methods for energy planning problems, Renewable and Sustainable Energy Reviews, 11, 1584-1595.

Mojtahedi, S.M.H., Mousavi, S.M., \& Makui, A., (2010). Project risk identification and assessment simultaneously using multi-attribute group decision making technique, Safety Science, 48(4), 499507.

Opricovic, S., \& Tzeng, G.H. (2004). Compromise solution by MCDM methods: a comparative analysis of VIKOR and TOPSIS. Eurpean Journal of Operational Research, 156, 445-455.

Pao, H.-T. , \& Fu, H.-C. (2013). Renewable energy, non-renewable energy and economic growth in Brazil, Renewable and Sustainable Energy Reviews, 25, 381-392.

San Cristóbal, J.R., (2011). Multi-criteria decision-making in the selection of a renewable energy project in spain: The Vikor method, Renewable Energy, 36, 498-502.

Vahdani, B., Mousavi, S.M., Hashemi, H., Mousakhani, M., \& Tavakkoli-Moghaddam, R., (2013). A new compromise solution method for fuzzy group decision-making problems with an application to the contractor selection, Engineering Applications of Artificial Intelligence, 26, 779-788.

Wang, B., Kocaoglu, D.F., Daim, T.U., \& Yang J., (2010). A decision model for energy resource selection in China, Energy Policy, 38, 7130-7141.

Yeh C.-H. , \& Chang,Y.-H., (2009). Modeling subjective evaluation for fuzzy group multicriteria decision making, European Journal of Operational Research, 194, 464-473. 